\section{Olfaction goes digital}

Neural circuits that encode sensory inputs must be sensitive to small changes in the quality of a stimulus but must also be able to identify certain stimuli as identical despite minor variations caused by background 'noise'. A neural network could achieve this by classifying sensory inputs into discrete patterns of output activity. Now, Niessing and Friedrich show that the activation patterns of mitral cell populations in the olfactory bulb change abruptly in response to gradual variations in the quality of an odour but are not affected by

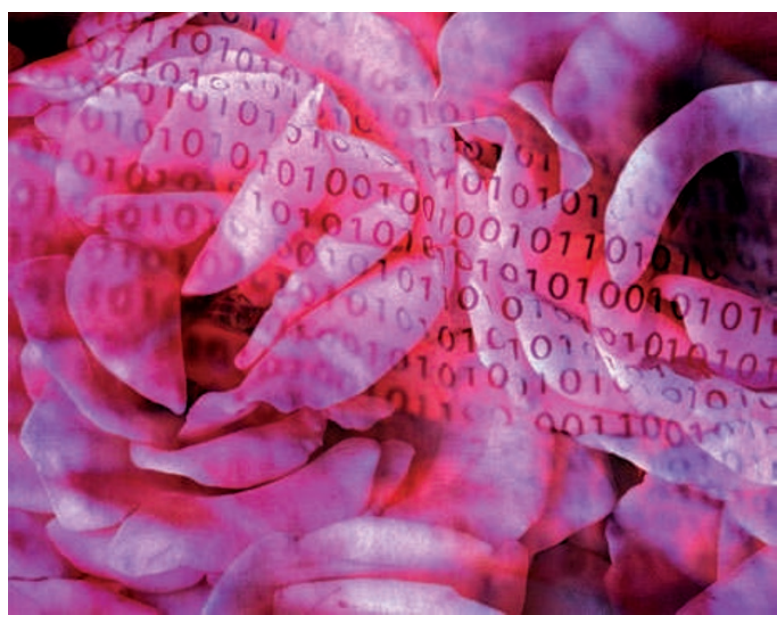

changes in odorant concentration, suggesting that the first processing step in odour recognition takes place at this level.

The authors measured changes in the firing rate across large populations of output neurons in the olfactory bulb of adult zebrafish using the novel technique of temporally deconvolved two-photon $\mathrm{Ca}^{2+}$ imaging. Although on average the firing rate of mitral cells increased with increasing odorant concentration, the pattern of activity across the population remained constant. By contrast, exposure to odorants with different molecular structures evoked different activity patterns in the same neuronal populations, suggesting that these patterns represent odour identity rather than intensity. To determine the minimal difference between two odorants that results in separation of neuronal activity patterns, the authors gradually changed the concentration ratio of two different odorants and used intermediate mixtures to stimulate the explants. The activity patterns evoked by these mixtures did not change gradually, but exhibited an abrupt transition at a certain threshold. When the authors mixed odours with different characteristics, they observed novel activity patterns that were separated by discrete transitions and had not been previously evoked by the pure components. Importantly, these abrupt transitions cannot be observed if the analysis is limited to individual mitral cells.

These results are in agreement with several behavioural observations, including the finding that animals have the ability to perceive odour mixtures as distinct from their components and fail to recognize a background odorant when it is underrepresented in a mixture (masking). This odour categorization imposes a limit on the resolution of the olfactory system, but at the same time enables the system to tolerate a certain amount of input noise. Signal processing at the level of the olfactory bulb may therefore be an important first step in odour perception that precedes later cortical computations.

Cristian Bodo

ORIGINAL RESEARCH PAPER Niessing, J. \& Friedrich, W. Olfactory pattern classification by discrete neuronal network states. Nature $\mathbf{4 6 5}$, 47-52 (2010) 